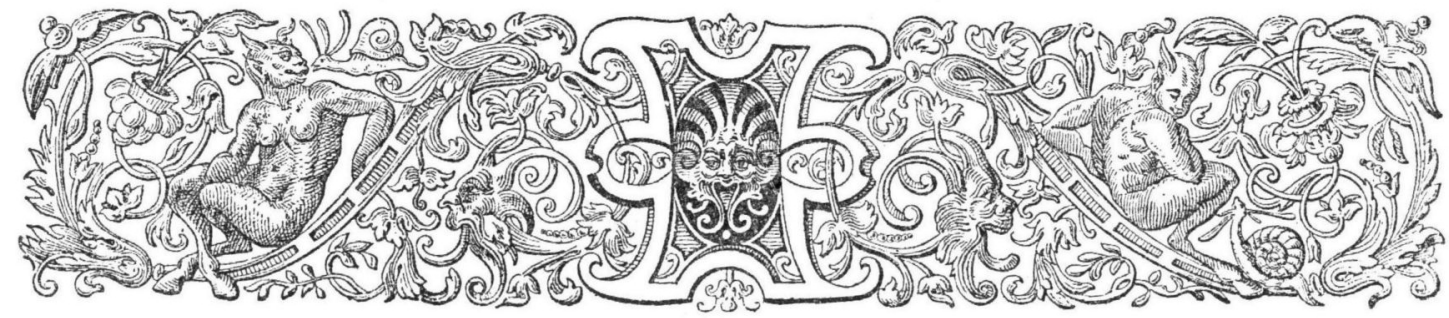

DIE BIBLIOTHEK

DES

\title{
PRINZEN MORITZ VON ORANIEN \\ VON
}

Dr. ANTON CHROUST.

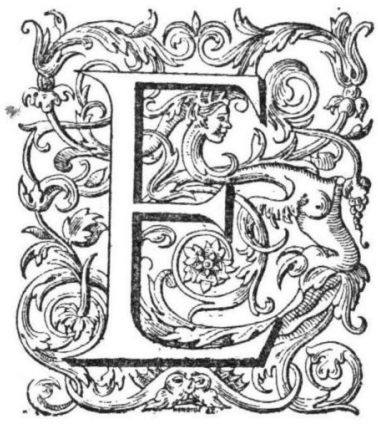

IN freundlicher Zufall hat uns das Verzeichnis der Büchersammlung des Prinzen MORITZ von ORANIEN erhaiten. Ich fand es gelegentlich meiner Arbeiten für die Lebensgeschichte des ABRAHAM, Burggrafen und Herrn zu DoHNA ${ }^{1}$ ), in dem Familienarchiv der gräflichen Familie Dohna zu Schlobitten in Ost-Preussen (Fasz. 4I/2). Dieses für die Geschichte des ausgehenden XVI. und des ganzen XVII. Jahrhunderts ungemein reichhaltige Archiv bietet bei den engen Beziehungen, durch welche die Herrn zu DoHNa geraume Zeit mit den Niederlanden verbunden wurden, überhaupt eine Reihe sehr beachtenswerter Mitteilungen in Tagebuichern und Briefwechseln für die politische und für die Geistesgeschichte der Vereinigten Niederländischen Provinzen.

1) Abraham von Dohna. Sein Leben und sein Gedicht auf den Reichstag von 1613 . München, I8g6. 
Für die DoHNA nicht minder wie für den grössten Teil des reformierten Adels Deutschlands bedeuteten die Niederlande die hohe Schule der Kriegskunst; unter MORITZ von ORANiEN oder Graf Wilhelm LuDwig gedient zu haben, war geradezu Bedingung für alle jene, die später in ihrer deutschen Heimat nach höheren militärischen Ehren strebten; es gibt fast keinen Fürsten der spätern protestantischen Union, noch einen hõheren Befehlshaber derselben, der dieser Forderung nicht genügt hätte. - Noch ein Zweites verband die Niederlande mit dem reformierten Deutschland: das reformierte Kirchenwesen, dessen Entwicklung in den Provinzen man diesseits des Rheines mit grösster Spannung verfolgte. Neben den militärischen und theologischen lockten aber auch die allgemeinen Bildungsinteressen nach Leyden und Gröningen, nach Amsterdam und dem Haag; die Kavalierstour umgieng, namentlich seit dem Beginn des XVII Jahrhunderts, nicht leicht die Provinzen; mit den Grössen der Leydener Universität Briefe zu wechseln gehörte für die gebildeten Kreisen des reformirten Deutschlands zum guten Ton; die deutsche Literatur weist nicht spärliche Spuren der Beeinflussung durch die niederländische auf; auch auf Tracht und Sitte haben diese Beziehungen des nördlichen Deutschlands zu den Niederlanden merkbar eingewirkt; es hat eine Zeit gegeben, wo der vollendete deutsche Edelmann sich wie ein holländischer Mijnheer zu geben hatte.

Unter den Kulturvermittlern zwischen Deutschland und den Niederlanden sind die Brüder Dohna zweifellos nicht die letzten gewesen: drei von ihnen, FabIan, Abraham und DiETRICH, haben längere oder kürzere Zeit unter dem Oranier gegen die Spanier gefochten; ein vierter, CHRISTOPH, der erst verhältnismässig spät, nach der Katastrophe des kurpfälzischen Hauses, nach dem Haag kam, wo der Winterkönig Hof hielt, ist sogar in nahe verwandtschaftliche Beziehungen zur oranischen Familie getreten; Prinz FRIEDRICH HeInRICH vermählt sich mit Amalia Gräfin von Solms-Laubach, der Schwester der Gemahlin Christophs ZU Dohna; dank dieser Verwandtschaft wurde Christoph ZU DohNA Generalgouverneur der Orange. Im Dienst des Winterkönigs hat auch der fünfte Brüder ACHAz wiederholt und lange sich im Haag aufgehalten.

Aelter sind die Beziehungen Abrahams zu Dohna zu den Niederlanden und zu MORItz von Oranien; von 1604 bis I609 hat Abraham nahezu ununterbrochen in den Niederlanden geweilt, - wie seine tagebuchartigen Etntragungen in die Schreibkalender dieser Jahre erkennen lassen - meist in unmittelbarer Nähe des Statthalters. Mit diesein machte er die wenig glücklichen Feldzüge von 1605 und 1606 mit; an den langwierigen Anstands-Verhandlungen hat ABRAHAM häufig als Ohren- und Augenzeuge teilgenommen; was er tiber deren wechselvollen Verlauf Tag für Tag in seinen Kalender einträgt, dürfte wol zu dem 
zuverlässigsten gehören, was über diese Dinge uns mitgeteilt wird, und verdient durchaus die Aufmerksamkeit der Geschichtsforscher.

Nicht nur die militärischen, auch die wissenschaftlichen Interessen des hochgebildeten Oraniers hat der strebsame und lernbegierige Ostpreusse geteilt. Die Zeiten unfreiwilliger Musse während der Verhandlungen hat ABRAHAM zur Ausfüllung der Lücken in seinem Wissen verwendet; nicht nur mit der Landessprache, auch mit dem spanischen und englischen machte er sich in Haag bekannt. Er verkehrte mit den leydener Theologen und Philologen, die im Hause des Statthalters aus und ein giengen. Sein Hauptinteresse gehörte allerdings der angewandten Mathematik, zu der Prinz MORITz, der auf die Theorie der Kriegskunst selbst grosses Gewicht legte, ABRAHAM Aneifrung und Anleitung gegeben zu haben, scheint, vor allem dadurch, dass er ihm seine, besonders für die Kriegswissenschaften reichhaltige Büchersammlung zur Verfügung stellte. Wie eifrig Abraham diese Erlaubniss benützte, zeigt, dass er im Mai 1609 einen Katalog der oranischen Bibliothek zusammenstellte und später noch ergänzte.

Dieses Bücherverzeichnis, das ich einem Wunsche der Herrn Herausgeber entsprechend im folgenden zum Abdruck bringe, gibt Zeugnis von den vielseitigen Interessen des Statthalters; dass die Kriegswissenschaftliche und die historische Literatur die theologische an Zahl erheblich übertrifft, wird nicht überraschen, muss aber doch hervorgehoben werden. Theoretische und angewandte Mathematik neben der Architektur nimmt einen breiten Platz ein.

An politischen und theologischen Streitschriften war die Bibliothel nicht reich, eifriger wurde die Literatur der ritterlichen Künste gesammelt; neben den Werken über Pferde, Falken, Hunde, stehen auch Gesners zoologische Werke. Von den geheimen Wissenschaften, Alchemie, Magie, Daemonologie scheint MorITz wenig gehalten zu haben. Am meisten. Beachtung verdient die Abteilung Miscellanea, bei welchem Schlagwort auch die schöne Literatur untergebracht ist; zahlreich ist sie allerdings nicht, doch die Werke des RAMUs und RONSARD sind vorhanden. Fleissig scheint der Prinz dagegen Kupferstiche gesammelt zu haben.

Die Bibliothek zählt im ganzen 403 Werke in 432 Bänden; manches Werk ist doppelt vorhanden, manches in mehreren Sprachen. Natürlich überwiegen die Werke in lateinischer Sprache; sie machen ungefähr die Hälfte der ganzen Sammlung aus. Ein starkes Fünftel ist in französischer, etwa ein Sechstel (64) in holländischer Sprache abgefasst, Werke in italienischer Sprache zählte ich etwa 30, die deutsche Sprache bringt es kaum auf ein Dutzend, die spanische Literatur ist durch drei Bücher, die englische gar nicht vertreten. Eine genaueve Statistik ist bei der geringen Sorgfalt, die ABRAham von DoHna der Wiedergabe der Titel angedeihen liess, nicht wol zu geben. 
Im Anschluss teile ich noch das Verzeichniss der Bücherschätze mit ${ }^{1}$ ) die der vorhin erwähnte DIETRICH vON DoHNA, der jüngere Bruder AbRahaMs, bis zum Jahr I 616 für sich erworben hatte. DiETRICH, der 1620 als kurpfälzischer Oberstleutnant bei Rakonitz fiel, hat einen grossen Teil seines Lebens in niederländischen Diensten und gleichfalls in der Nähe des Prinzen MORITZ zugebracht. Es ist die Bibliothek eines Reitersmannes, aber eines frommen; Bibel und Psalter stehen neben der Silva curiosa und dem Ingenioso Hidalgo von der Mancha; im Gegensatz zur Bibliothek der Statthalters besteht DIETRICHS Büchersammlung fast zur Hälfte aus spanischen Werken, dagegen ist die lateinische Sprache gar nicht vertreten, denn der Rittmeister dürfte auch den Plutarch in französischer Uebersetzung gelesen haben; freilich hat ihm auch die deutsche Literatur fast nichts zu bieten gehabt. Für die geistigen Interessen jener Zeit scheint mir auch dies kleine Verzeichniss nicht ohne Bedeutung zu sein.

I.

MAURICII PRINCIPIS CATALOGUS.

Abraham von Dohna. I608, 24 April. Hagae.

G. W. G.

HISTOIRES.

Histoires anciennes tant Grecques que Latines.

In Folio.

T. Livius.

NICEPHORI historia ecclesiastica. Julius Caesar; latine.

XENOPHONTIS opera omnia.

5. Numismata.

C. Tacitus, Lipsir.

ZONARAS, NICEPHORUS.
Polibius gallice.

Julius Caesar GolziI.

Io. Caesar Augustus eiusdem. Fasti magistratuum Romanorum. Eusebius.

Strabo.

THuCIDIDES.

I5. Suetonius.

POLYBIUS graece et latine.

De antiquitatibus Romanis.

Q. Curtius.

1) Schlob. Arch. Fasz. 47/2. 
HERODOTUS.

20. De ludis Circensibus.

Les fastes.

$$
\text { In } Q \text { uarto. }
$$

Commentarii in CAESAREM Ital. PALladil.

Suetonius gallice.

Der Grieken opgang ende ondergang. 25. LIPSII admiranda.

In Octavo.

$$
\text { In } 12^{\circ} \text {. }
$$

ARRIANUS.

Imagines imperatorum.

HISTOIRE MODERNES.

In Folio.

Tevet, des hommes illustres.

SCALIGER, de emendatione temporum.

30. Chronologia Henrici Bunting.

Comminaeus, gallice.

Navigationes de JEAN BAPTISTE

RAMUSE ital. tom. I, tom. 2, tom. 3 .

PIfTer Bor, neederlandsche historie I. deel, 2 , deel.

Chronique ancienne et moderne de Hollande et Zeelande, tom. I, tom. 2 .

35. Memorie neederlantscher historie.

Description des Pais-bas, GuICC. [IARDINI].

Chronike von Hollant.

EMANUEL DE METEREN, neederlandtsche historie.

Historiae Sabellicae tom. I, tom. 2, tom. 3 .

40. Jovius.
Description des royaulmes du monde.

Platina, de vitis pontificum.

Chronica de Carlo V. hispan.

45. Annales Austriacorum principum GERARDI DE RoO.

Genealogia HenNingII.

GUICCIARDINI, description des Paisbas, nouv. edition.

Historischer bericht der braunschweigschen sach.

In $Q u a r t o$.

Relazione del reame di Congo.

50. Discours de la guerre d'Allemagne.

Vitae et icones sultanorum cum historia Pannoniae.

Belegerung von Alkmaer.

Histoire de la paix Maltien(?).

Beschrieving van die staaden van Hollant van GODEFROY BOOT.

In Octavo.

55. Republique van Schwitzerlant.

Historie van Westindien, JosephUS DE Acosta.

Aventure de roy SEbastian.

Speculum tyrannidis regis Hispaniae.

Cronicon CARIONIS.

6o. Guicciardin, des guerres d'Italie, I. volume, 2. volume.

Histoire de l'estat de France.

Commentaires de MonLuc.

Inventaire de France . partie, 2. partie, 3 partie.

Memoires de la Ligue I. volume, 65. Historia Basilidis Moscovitae.

Verradereye van den jesuiten GARNET tegens den Koning van Engelant. 


\section{DE LA GUERRE.}

De arte militari.

In Folio.

LEONART FroNSBERGER.

Vom Keyserligen rechten.

Aelianus, latine.

70. $\quad "$ gallice.

ONOSANDER,

LEO IMPERATOR.

Paralleli militari di PAtricio, I. parte, 2. parte.

Castrametation de CHOULx.

75. Instructions de la guerre.

Devoli et BRANCACIo, dell'ordonanzie et battaiglie.

De la discipline militaire.

VALTURIN, de l'art militaire, gallice.

Della militare disciplina, de CIMt .. SANESE.

80. Harangues militaires.

GEORGIT AGRICOLA, de arte militari.

$$
\text { In quarto. }
$$

LIPSIUS, de militia Romana.

PATRICIUS, de militia Romana.

Dell'espugnationi et diffese di GABRIEL BusCa.

85. Delle cose di guerra.

Discorso del capitan CHRISTOVAL LOCHuGA.

Instructien voor jonge soldaten te voet ende te paerd.

In octavo.

Antonius Vulturinus Jesuita, de re militari.

9o. Milice françoise de Mommorancr.

Polieni stratagemata.
ONOSANDER, de re militari.

LEO IMPERATOR, de apparatu bellico.

Ramus, de militia Caesaris. 95. Spiegel der oorlogsaken.

DES FORTIFICATIONS.

In Folio.

Fortification d'ADRIAN CONFLANS.

Fortification de Galasso.

Carlo Tetti.

PASSINO.

Ioo. Fortification de Jacques Perret.

LORINI.

SPECKLE.

WINKELMANS discours des fortifications.

$$
\text { In } Q \text { uarto. }
$$

GABRIEL BUSCA, des fortifications.

105. Catanets, latine.

$$
\text { "gallice. }
$$

Sterkten Bouwing, Simon Stevis. Le guide des fortifications.

ARCHITECTURE ET MACHINES.

In Folio.

Machine di Ramelli.

IIO. Theatrum instrumentorum BESSONII.

Architecture di JaC. BAROSSIO.

Regole de'cinque ordini di architectura eiusdem.

Vitruvius, latine.

$$
" \text { gallice. }
$$

I I 5. Theatrum Ressonir [?] Termes de Huges Sambin. Luigi Collado, de l'artigleria. 
Modelles d'artifice du feu de JoSEPH BAILLOT.

La pyrotechni.

120. Architectura de Pietro Cataneo.

In Quarto.

La pyrotechnie.

Machine et quartieri.

M. Johan Bouvier, von der buchsenmeisterei.

Poliorcetica LIPSII.

I25. Essamini de' bombardieri.

Le opere di TARTAGLia.

MATHEMATICA.

Arithmetica et Astronomica.

In Folio.

Astronomicum Caesarium.

Opus Palatinum. '

Durer, de la proportion.

I 30. Sebastian Munster und Albert

DürER.

ARCHIMEDES.

Adrian Romanus, in A rchimedem.

TyChonis BRAHE, stellarum errantium restitutio.

Eiusdem Astronomica.

I35. Gnomonica Clavit.

Ludolf vaN Coelen, van den cirkel.

Prodiadochi in I. librum EucLIDIS.

CARdanus, de regulis algebraicis.

$n$ de astrorum iudiciis.

r40. Opuscula mathematica OrontII

FinaE.

De numeris perfectis.

Tabula generalis JOANNIS REgIOMONTANI FRANCI.

Oud-Holland 1897 .
Astrologiae methodus JoANNis Garcani.

Ptolomaei Almagestum.

145. Speculum uranicum.

Scaliger, de quadratura circuli.

CARDANUS, de rerum varietate.

Wiskonstige gedachtenissen daer

hem S. Exc, in geoefnet heeft

door Sim. Stevin.

In $Q$ uarto.

Entechna musica.

I5O. Tychonis Brahe, Phenomenis.

Aupolicus, de sphera.

Apologia ClaviI.

Almanach perpetuel.

Ephimerides Martini Everardi.

I55. Heronis spiritualium liber.

Traicté de toiser.

Weegkonst Stevins.

Theorica nova planetarum.

Arithmetica StIfelif.

I60. Conformatio horologiorum WITEKINDI.

CARDANI tractati varii.

Cosmolabe de Jacques Besson.

De elementis et orbibus coelestibus Messahala.

Tabulae Prutenicae motuum caelestium per ERASMUM REINHOLT.

I65. Primus liber tabularum directionum per eundem.

WERnERUS, de elementis.

Tabulae resolutae JOANNIS VERDUNGEN.

Calendarium Petri Pilati VeroNENSIS.

Enchiridion artis numerandi. 
I7o. Compositio quaerentis novi.

CARDANI mathematici libelli. Opuscula mathematica PETRI DE Alliaco.

ARCHIMEDEs, de incidentibus.

De proportione orbium coelestium JOANNIS KEPLERI.

175. Tractatus arithmeticus partium et allegationumPETRI DESALMACO.

- Ephemerides JOSEPHII MOLETII mathematico.

Clavius in sphaeram JoANNIS DE Sacrobusto.

In Octavo.

Arithmetica Gemmae Frisir.

ADRIANI ADRIANIDIS doctrina sphaerica.

i 80. Josephi SCALigeri elenchus per Clavium.

GEOGRAPHICA ET COSMOGRAPHICA.

In Folio.

C. Ptolomaei opera.

Geographiae liber manuscriptus.

Pauli Merulae cosmographia generalis.

Ptolomaei tabulae.

I85. Theatrum urbium orbis terrarum. tomus I, 2, 3, 4, 5 .

Urbium theatrum [tomus 5.] GEorgit Praun.

MERCATORIS cosmogra phia.

Atlas Mercatoris.

Cosmographia ORTELII.

190. Cartenboek der mitländischer zee.

Reisboek der Portugalloisen door LINSCHOTEN.
Carten ende verbalen belangende Zeelant.

Napasser der oost- ende westzee.

Enkhuyser zeekartenboek von Lucas Wagenaer.

195. Americae et Floridae descriptio I et 2.

Americae pars 3 et 4 .

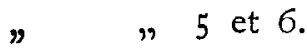

$" \quad 7$ et 8 .

$" \quad 9$

Augmentum Ptolonate de novo orbe.

In $Q$ uarto.

Cosmographia Appiani.

Geographiae Ptolomaer pars II. Terrae totius descriptio.

200. Portuum investigandorum ratio Grotir.

Jan Huygens Linschoten.

Syntagma ARateorum.

Joannis Eliotae Angli Hidrographia.

Konst van zeevart.

205. SimON BARENTS beschreibung von Nova Zembla.

Liecht der zeevart door WiLlem Janson.

Tresor van die zeevart.

Reysen van SPILbERGEN.

T' Goult-coningryk van Guinea.

2 Io. Zachiariis Silius, de situ orbis. Theatrum mundi et temporis Jo. anNis Pauli Gallucit.

In Octavo.

Historie van die Westindien JoSEPHI D'ACOSTA. 
Reyse van Houltman in t' eiland van Sumatra.

\section{GEOMETRICA.}

$$
\text { In Folio. }
$$

Geometria DURERI.

215. Manière pour mesurer les longitudes par WiLh. NAUTON.

Chordarum resolutio ADRIANI de arcubus Romanis.

Resolutiones cordarum.

Summa dearithmetica etgeometrica proportione.

Propositiones Ptolomaei de sinibus et coreis. Georg PERTaG (PEUERbach)

220. Instrumentum sinuum PETRI ADRIANI.

Demonstrationes JACOBI PolataRII in EUCLIDIS elementa geometrica.

De triangulis omnimodis JoANNIS REgiomontanj.

$$
\text { In } Q \text { uarto. }
$$

Theorie du quadrant.

Pratique de la geometrie d'ORONCE.

225. Practike der lantmetery.

Discobrimientos geometricos.

6 Boeken EuCLIDIS van der gront der lantmeterye.

3 Tractat der mechanischen instrumenten L. HULSII.

Descriptio locorum solius regulae adminiculo manuscripta.

230. Wilebordi SNELliI geometria.
THEOLOGICA.

In Folio.

Biblia gallica.

Biblia hispanica.

Ecclesiasticus manuscriptus.

In $Q$ uarto.

Discours de la religion.

235. Tafereel von St. AldEgOndE.

Paraphrasis Gerobuli in Psalmos.

Junius in Danielem.

Tableau.

Utlegging des catechismi CORNE

LIS Corstens.

240. Staet der kerken CRISPINI.

L'estat de l'église TAFFIN.

Traicté de l'amendement de vie TAFFIN.

Oorsprong der tempeln RoDOLFI

Hospiniani.

Hugonis GrotiI pontifex.

245. Religionis libertas (tweemael).

Censura ubiquitatis JoANNIS LAMPADII.

Bible escrite à la main.

Meditation sur l'oraison de Jesus.

In Octavo.

Bible françoise.

250. Aurea salutis catena.

Sermon surla resurrection de Christ.

Wederlegging zeker geschrift JACOB JANSEN Herdooper.

Catechismus Witebergensis.

Uncti Jesus effigies.

255. Medication sur l'oraison de Jesus.

Hierosticon B. A BARLICOM. 
Responce apologetique du Sr. de $\mathrm{S}^{\mathrm{T}}$. AldEgonde.

De conjugio spirituali inter Chris. tum et ecclesiam.

Dos trattados del papa y della messa.

260. Pseaume de $S_{\text {T. Aldegonde. }}$

Oorsprong der herdoper secten.

Van die predestinatie van $M$. Pieter Wyrs.

Loci communes.

Explications des evangiles de MELANTON.

265. Clavis prophetica Caroli Galli.

Bulle van CLEMENS den 8. paues

Christliger extract aus den 4 evan. gelisten.

De papatu Romana carmen.

Confession de l'electeur FrEDERICQ 3.

270. Jesus Christ mit zynen aposteln beschriven door JOAN WIRYX.

In 120.

Pseaumes de David escrist par Ester Angloise.

INNOCENTIUS PAPA, de contemptu mundi.

\section{PHILOSOPHICA.}

In Folio.

SyDRACH.

Opuscule de Plutarcil I, 2.

275. Les essais de Montagne.

Vicissitude des choses.

El conde Balthasar CASTIGLIONE.

Cardanus, de subtilitate.
Ulissis ALDROVANDI philosophi medici ornitologiae tom. I, tom. 2 . 250. Eiusdem de animalibus et insectis. MARC Aurìle.

Hyppolitu's Salvianus, de aquatilibus.

Vogelbuch GESNERI (tweemael).

Tierbuch GESNERI.

285. Fischbuch Gesneri.

De avibus.

In $Q$ uarto.

De natura magnetis.

Phisionomia PORTAE.

ARISTOTEles de mundo.

290. Henrici Muthanae.

Liber secretorum VON DER HORST.

In Octavo.

Magia naturalis.

MEDICA ET CHIRURGICA.

$$
\text { In Folio. }
$$

AMbroise Paré.

Historia plantarum CLUSII.

In $Q$ uarto.

295. Heurnius, de morbis pectoris.

In Octavo.

De curandis venenis $I, 2$.

Apologia chimica JosePHI MICHELII.

Observationes FORESTII liber 2, liber 3 , liber 4 .

\section{MISCELLANEA.}

In Folio.

Calepinus undecim linguarum. 300. BOCACE, des nobles malheureux, Tuyn, helpt mi stouten.(?) 
In $Q$ uarto.

Epistres des princes.

Auriacum HeinsiI.

GROTII scutum Auriacum.

305. Oratio panegyrica Gertrudisbergen.

SCAligeri epistolae de vetustate gentis suae.

Incompste van den prins van Orang.

Apologie van den prins. "

Oratio panegyrica van der innemen van Groningen.

3Io. Victorie van die General-Staten. Princeps Auriacus.

Sonnets de M. JEAN PETIT.

Oratio panegyrica de militia.

Oratio panegyrica de victoria Turnhaltana.

35. Epitalamium comitis ab HoHEN [LOIIE].

Certamen novem Musarum.

Mauritius Alberti Wito.

Oratio panegyrica.

Epicedium ad principem MAU. RITIUM.

320. Oratio panegyrica Kinscirotr.

Mirabilia anni 1600.

Oppugnatio urbis Bredanae.

Bataille de Nieuport.

Gratulatio victoriae MAURITII adversus Albertum.

325. Triumpfliet.

Parenesis Belgicarum provinciarum.

Le miroir de l'union Belgique.

Utlegging van metamorphosis

Ovidii.

Grammatica CorneliI VALERII.

330. Cornel.rs BoIsens schryfboek.
La venerie et fauconerie de JAC. FouILLOUX.

Speelen van Haarlem door ZACHAR. HEINS.

Verbeeterung der schriftuer door Coel.haAs.

In Octavo.

Res gestae HeNRICI Burbonil.

335. Belgica demologia.

Martianus Capella.

Gesta Nassavica.

Savoisienne.

Origo principis MAURITII.

370. Philomela.

Florilegium epigrammatum.

Decameron de BOCACE.

Retorica VALERII.

Spraekboek malaisch.

345. Comedie van den loop deses tyts.

La venerie de l'empereur FREDERICQ.

BAUDII poëmata.

In Duodecimo.

Histoires tragiques I. 2. 3 .

Serrees de Guille. Bouchar.

350. Les devises heroique:

Oeuvres de Ronsart.

Grammatica RAMI.

Lettres PASQuiER.

Lettres du TRONChET, secretaire de la royne mère.

\section{L'ESCUYERIE.}

$$
\text { In Folio. }
$$

355. Von Zeumen.

La broue.

Anatomia del cavallo.

Infirmità del cavallo. 
Ritterlige reuterkunst und turnierbuch.

360. Peerdebeelder gedruckt.

Cavallo frenato di Piero ANTONIO Fetrato Napolitano.

In $Q u a r t o$.

LAURENT RUSE.

Frederigo Grigionf, ital.

Gloria del cavallo. gallice.

365. Claudio Coste.

Medizinboek van paerden van

M. JAN VAN DELfT.

Notitie van die generatie van paerden van zyn Exc. staende voor Ryswik.

Cesare Fiasisy.

$$
\text { In Octavo. }
$$

De' marchi de' cavalli.

L'E S C R I M E.

In $Q$ uarto.

370. L'escrime d'Achille Morozzi.

L'escrime de S. DEDIERE.

PETER BAILli.

Cabinet d'escrime.

Della scienzia della spada.

In Octavo.

375. Della scienzia della spada.

\section{PEINTURES.}

In Folio.

Effiges des princes et capitaines de nostre temps.

Imitationes MrCHaELIS ANGELI.

Beeldboek van koperdruksels.

Beelden.

380. Krichsbeelden.
Omnium gentium habitus' Joos VAN Boscher.

Ducum imagines.

In $Q$ u arto.

Gront der schilderkunst.

Gedenkboek van JACOB DUYM.

385. Perspective VRedeman. I. 2. teyl.

Perspective DE KOK ende VRIESE.

Perspective de SirigutTi.

Rítratto di Ioo capitani di guerra con gli lor fatti da Filippo Tomasino.

POLITICA ET JURIDICA.

$$
\text { In Folio. }
$$

Notitia dignitatum.

390. Poli[ti]que de Henry George LaUTERBACH [?]

Statuta hospitalis Hierosolimitani.

Tronus iustitiae (tweemael).

Placaeten ende ordonanz op t'stuck van die wildernisse.

In $Q$ uarto.

Politica LIPsIl (tweemael).

395. De iure belli Belgici.

Ordonance sur le faict de la justice.

Admonitio ad orbis terrae principes.

De feudis iuris scripti Hollandici. Credenzschreiben und instruction herzog CARls von SUdermanLAND.

400. Nic. Cragius, de repub. Lacedaemon.

In Octavo.

MACHIAVELLI discours.

Politica Danaet.

Edictum reginae Angliae. 
II.

\section{DIE BIBLIOTHEK DES RITTMEISTERS DIETRICH VON DOHNA IM JAHRE I6I6.}

Dos tratados del papa, el secundo es de la missa, in $8^{\circ}$.

La silva curiosa Julian DE MEDRANo, cavallero Navarro, in $8{ }^{\circ}$.

$\mathrm{El}$ ingenioso hidalgo Don Quichotte de la Mancha, in $8^{\circ}$.

Libro de los commentarios de Gato Julio Caesare, in $8 \circ$.

5. 2 Tomi vitarum Plutarchi, in $80^{\circ}$.

Dictionarium teutsch und franzö-

- zisch, in 8 .

La bible françoise, in $8 \circ$.

Histoire du grand TaMerLanes, in 80 .

La famosa y temeraria compania de Rompe Columnas.

IO. Les oeuvres de Cornelius Tacitus et deVElleius Paterculus, in $4^{\circ}$.
Tesoro de las tres lenguas francesa, italiana $y$ espannola, in $4^{\circ}$.

Christliche warnung des trewen Eckarts, in $8^{\circ}$.

Historie de Georges Castriot, in $8^{\circ}$.

Tragicomedia de Calisto y Melibea, in $12^{\circ}$.

Le theatre du monde, in $12^{\circ}$.

Des saines affections, in $16^{\circ}$.

Refraneso proverbios espanoles, in $8^{\circ}$.

Les oeuvres poetiques, in 160 .

I Psalmbuchlein franzözisch, in 160 .

I " niederlandisch, -

De la vida de Goncalo HernanDEZ, in $8_{0}$.

BARLAMONT niederlandisch und

- franzözisch, in $8^{\circ}$.

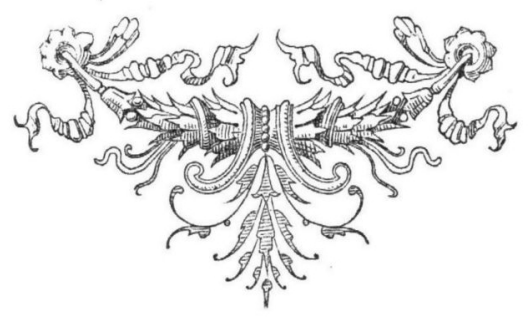

